\title{
CS Research Square \\ Review Adult Upper-limb Forearm Cystic Lymphangioma (Cystic Hygroma): A Case Report
}

\author{
Kai Cui \\ China Medical University \\ Yingkang Zhu \\ China Medical University \\ Fatai Lu \\ China Medical University \\ Rui Wang \\ China Medical University \\ Haifei Zhang \\ China Medical University \\ Zunpeng Liu ( $\nabla$ zpliu@cmu.edu.cn ) \\ China Medical University
}

\section{Case report}

Keywords: Cystic lymphangioma, upper extremity, D2-40, radical resection

Posted Date: July 26th, 2021

DOl: https://doi.org/10.21203/rs.3.rs-726806/v1

License: (c) (i) This work is licensed under a Creative Commons Attribution 4.0 International License.

Read Full License 


\section{Abstract}

Background: Cystic lymphangioma that is the most common type of lymphangioma, but it rarely occurs in the forearm. It may show localized invasive behavior, but is benign.

Case presentation: A 42-year-old woman was admitted to the hospital because she had discovered a right forearm mass approximately 3 years earlier.Ultrasound had shown a subcutaneous hypoechoic strip on the right forearm (nature to be determined). Magnetic resonance imaging (MRI) showed characteristics of a lymphangioma with irregular strips, and surrounding irregular small blood vessels showed hypointensity or isointensity relative to the T1-weighted imaging (WI) signals for nearby muscle and hyperintensity on T2WI.Hematoxylin-eosin (H\&E) staining showed a dilated cystic space lined with flat endothelial cells, and the cyst wall was rich in mature lymphocytes, smooth muscle, and irregular fatty small cysts.Immunohistochemical (IHC) staining of monoclonal antibody D2-40-labeled cystic lymphangioma endothelial cells was performed and showed focal expression of D2-40.

Conclusions: The atypicality of clinical symptoms and imaging findings makes preoperative diagnosis difficult, so histological examination is necessary for the diagnosis of cystic lymphangioma. At present, if the disease affects the function or appearance of the body, the ideal treatment is radical resection to reduce recurrence. Incomplete resection causes relapse of the disease.

\section{Background}

Lymphoma hygroma, also known as cystic lymphangioma, is a rare congenital malformation composed of lymphatic and lymphoid tissues. The atypicality of clinical symptoms and imaging findings makes preoperative diagnosis difficult, so histological examination is necessary for the diagnosis of cystic lymphangioma ${ }^{[1]}$. Cystic lymphangioma, although it may show localized invasive behavior, is benign, and subsequent development of a malignancy is very rare. If these lesions are not completely removed, they may recur locally ${ }^{[2]}$. At present, if the disease affects the function or appearance of the body, the ideal treatment is radical resection to reduce recurrence. Cystic lymphangioma is very rare and common in children. Approximately $95 \%$ of cystic lymphangiomas are found in the head, neck, and armpits, and the remaining $5 \%$ are found in the mediastinum and abdominal cavity, including the mesentery, retroperitoneum, and bone ${ }^{[3]}$. In a large series of studies on lymphangioma by researchers, such as Alqahtani, 23 (12\%) of 186 children had limb lymphangiomas, of which $11(6 \%)$ were in the upper limb $b^{[4]}$. Lymphangiomas can occur in adults, do not show up when they are young, and are usually secondary to a local trauma or infection. There are approximately 100 reported cases ${ }^{[5]}$. Lymphangioma of an upper extremity is rare in adults, and lymphoma of the forearm is even rarer. However, there have been a few reports recently. Here, we present the case of a 42-year-old adult woman who was diagnosed in April 2019 with a radial lymphangioma in the right forearm. The surgical resection was successful, and no recurrence was observed during 7 months of follow-up.

\section{Case Presentation}


The patient was a 42-year-old woman. She was admitted to the hospital because she had discovered a right forearm mass approximately 3 years earlier that gradually increased in size but did not cause noticeable pain. She had undergone an ultrasound examination without special treatment at another hospital 1 year before admission to our hospital. Physical examination: A 6×1-cm mass was observed on the right forearm on the temporal side of the iliac crest. The mass was tender and exhibited a smooth surface, good mobility, a tough texture, and a clear boundary with surrounding tissues. Ultrasound performed at another hospital had shown bar-shaped hypoechoic signals under the skin of the right forearm with a clear boundary and no signal indicating blood flow. Ultrasound also showed a subcutaneous hypoechoic strip on the right forearm (nature to be determined).

Magnetic resonance imaging (MRI) showed characteristics of a lymphangioma with irregular strips, and surrounding irregular small blood vessels showed hypointensity or isointensity relative to the T1-weighted imaging (WI) signals for nearby muscle and hyperintensity on T2WI. The widest point was approximately $1.0-\mathrm{cm}$ wide and $8-\mathrm{cm}$ long. The combined symptoms, signs and imaging evidence support the diagnosis of right forearm radial lymphangioma (Fig. 1).

The patient was given brachial plexus anesthesia before making a 7-cm long longitudinal incision above the right forearm tumor through the skin and each of the subcutaneous layers, which were separated bluntly. The 10-cm long mass was cord-like, swollen in many places, and accompanied by blood vessels supplying nerves. The proximal and peripheral adhesions were not tight. The distal end and nerve blood vessels (radial nerve branches and radial arteriovenous) had increased adhesion but could be separated normally. The distal and proximal ends touching normal tissue (lymphatic appearance) were careful peeled off and ligated separately (Fig. 2). The excision was sent for pathological analysis. The area was repeatedly flushed with normal saline, followed by hemostasis and suturing. A pressure dressing with excipients was applied. After the operation, she was returned to her ward. The postoperative section was cystic, thin-walled, and soft. After making an incision, clear, slightly yellowish, watery mucus flowed out.

Hematoxylin-eosin (H\&E) staining showed a dilated cystic space lined with flat endothelial cells, and the cyst wall was rich in mature lymphocytes, smooth muscle, and irregular fatty small cysts. No atypical cells were found (Fig. 3). Immunohistochemical (IHC) staining of monoclonal antibody D2-40-labeled cystic lymphangioma endothelial cells was performed and showed focal expression of D2-40 (Fig. 4).

\section{Discussion And Conclusions}

Lymphangiomas can be divided into three categories: cystic lymphangiomas or lymphadenomas, which contain large macroscopic lymphatic cystic spaces composed of collagen and smooth muscle; and cavernous lymphangiomas, which consist of buds that eventually form lymphatic vessels that develop with less space. Capillary lymphangioma, which consists of small lymphatic vessels, contains the smallest cystic space. Cystic lymphangiomas is the most common type of lymphangioma ${ }^{[6,7]}$. There are many reference mechanisms that may explain the pathophysiology of lymphedema. For example, embryologically, during the normal development of lymphatic vessels, lesions associated with 
lymphedema are thought to originate from isolated lymph tissue in the lymphatic sac, which cannot communicate with the rest of the lymphatic or venous system. Subsequent expansion of isolated lymphoid tissue leads to cystic morphology of these lesions ${ }^{[8]}$. Therefore, this mechanism can explain why there is a high probability of lesions in children but an extremely low probability in adults.

Lymphocytic tumors can occur in adults. Clinical symptoms usually appear in the form of soft or hard nodular masses of an unclear nature on the extremities that cause pain when under pressure, during movement, or with trauma but rarely cause pain when a person is not moving ${ }^{[5]}$. Ultrasound can detect such cystic masses with high sensitivity and is an ideal initial imaging examination method for tumors of unknown nature. It is readily available, relatively inexpensive, and does not involve ionizing radiation. Therefore, it can be used as the primary examination. The disadvantage is that it is too extensive and less specific for lymphoma. Cystic lymphangioma can appear as a multilobular mass with internal septations and anechoic fluid during ultrasonography, and the identification of intra- or intra-cystic echogenic intervals is an important factor in diagnosis ${ }^{[9]}$. MRI is a necessary condition for the diagnosis of cystic lymphedema at present. Relying on its improved soft tissue resolution and ability to differentiate different tissue types, MRI provides a more accurate description of cystic masses. It not only can explain the invasion of surrounding tissue by a lesion, but also provide indispensable information for selecting the surgical range. On MRI, depending on the contents of the capsule, a lesion can be shown as serous, chylous, hemorrhagic, or mixed. However, in general, lymphangiomas are hypointense or isointense relative to muscle on T1WI and hyperintense on T2W[10]. Histopathological examination is the "gold standard" for diagnosis of lymphoma and can be accurately diagnosed. H\&E staining can show dilated cystic spaces lined with flat endothelium and a cyst wall rich in lymphocytes, smooth muscle, and irregular small fatty cysts. However, H\&E staining is sometimes difficult for diagnosis of lymphoid tissue. IHC staining of cystic lymphangioma with monoclonal antibody D2-40 is more sensitive than H\&E staining, which can improve accuracy. D2-40 is a specific lymphatic endothelial marker ${ }^{[11]}$, which specifically stains lymphatic vessels and does not react with vascular endothelial cells ${ }^{[12]}$. At present, if the disease affects the body's function or appearance, the ideal treatment is radical resection to reduce recurrence. Incomplete resection is the only cause of relapsed disease ${ }^{[13]}$, and the probability of recurrence ranges from $0-13.6 \%[14]$.

In conclusion, lymphoma is common in children and rarely occurs in the forearms of adults. Clinical and imaging evaluations are necessary, but the final diagnosis is likely to be made by pathological evaluation of a resected specimen. Although complete resection of lymphangiomas can be a major challenge and may be associated with serious complications, lymphadenomas rarely resolve spontaneously, especially if they are symptomatic, and cause human physiological dysfunction. Radical resection is the main treatment. Regular follow-up is required after surgery to monitor a cyst for recurrence.

\section{Declarations}

\section{Ethics approval and consent to participate}


All procedures used in this research were approved by the the Fourth Affiliated Hospital of China Medical University Clinical Research Ethics Committee, and obtained informed consent from adult research participants.The Ehics Committee's Reference Number区EC-2020-KS-026.

\section{Consent for publication}

Not applicable.

\section{Availability of data and materials}

All data generated or analyzed during this study are included in this published article.

\section{Competing interests}

The authors declare that they have no conflict of interest.

\section{Funding}

Te study was funded by the General program of China Postdoctoral Science Foundation (2019M66166) and Liaoning Provincial Doctoral Fundation (20180540065) for the writing and publication of the manuscript.

\section{Authors' contributions}

Yingkang Zhu, Kai Cui and Zunpeng Liu designed the case report, analyzed the data, and wrote the initial draft of the manuscript. Fatai Lu and Haifei Zhang performed the research and analyzed the data .Rui Wang contributed with histologicalanalysis. All other authors have contributed to data collection and interpretation and have critically reviewed the manuscript.

\section{Acknowledgements}

Not applicable.

\section{References}

1. Thiam O, Faye PM, Niasse A, Seye Y, Gueye ML, Sarr IS, et al. Cystic mesenteric lymphangioma: A case report. Int J Surg Case Rep 2019;61:318-321. doi: 10.1016/j.ijscr.2019.07.051.

2. Garcia Dubra S, Vega Chaves A, Varela Romero JR, Mosquera Oses JJ. Cystic lymphangioma of the breast. Breast J 2019;25:523-525. doi: 10.1111/tbj.13269.

3. Nagano H, Kimura T, lida A, Togawa T, Goi T, Sato Y. Cystic lymphangioma in the peripheral jejunal mesentery in an adult and excision with laparoscopic-assisted surgery: a case report. World J Surg Oncol 2019;17:170. doi: 10.1186/s12957-019-1713-6.

4. Alqahtani A, Nguyen LT, Flageole H, Shaw K, Laberge JM. 25 years' experience with lymphangiomas in children. J Pediatr Surg 1999;34:1164-1168. doi: 10.1016/s0022-3468(99)90590-0. 
5. Rahal MJH, Abou Al Ezz MR, El Hajj RA, Chokor JZ, Nasser SM, Moucharafieh RC. Elbow Cystic Lymphangioma in an 8-Month-Old Boy. Case Rep Orthop 2019;2019:8762614. doi: $10.1155 / 2019 / 8762614$.

6. Wong KT, Lee YY, King AD, Ahuja AT. Imaging of cystic or cyst-like neck masses. Clin Radiol 2008;63:613-622. doi: 10.1016/j.crad.2007.12.007.

7. Azim MT, Hussain SM, Mughal MA. Primary Supraclavicular Cystic Lymphangioma in an Adult: A Rare Presentation. J Coll Physicians Surg Pak 2019;29:S11-S12. doi: 10.29271/jcpsp.2019.06.S11.

8. Mirza B, ljaz L, Saleem M, Sharif M, Sheikh A. Cystic hygroma: an overview. J Cutan Aesthet Surg 2010;3:139-144. doi: 10.4103/0974-2077.74488.

9. Zouari M, Ben Dhaou M, Kchaou R, Jallouli M, Mhiri R. Unusual sites of cystic lymphangioma in children. Arch Pediatr 2015;22:676-677. doi: 10.1016/j.arcped.2014.09.001.

10. Nguyen K, Siegelman ES, Tu W, Schieda N. Update on MR Imaging of cystic retroperitoneal masses. Abdom Radiol (NY) 2019. doi: 10.1007/s00261-019-02196-9.

11. Arigami T, Natsugoe S, Uenosono $Y$, Arima $H$, Mataki Y, Ehi K, et al. Lymphatic invasion using D2-40 monoclonal antibody and its relationship to lymph node micrometastasis in pN0 gastric cancer. $\mathrm{Br} J$ Cancer 2005;93:688-693. doi: 10.1038/sj.bjc.6602739.

12. Yonemura Y, Endou Y, Tabachi K, Kawamura T, Yun HY, Kameya $T$, et al. Evaluation of lymphatic invasion in primary gastric cancer by a new monoclonal antibody, D2-40. Hum Pathol 2006;37:11931199. doi: 10.1016/j.humpath.2006.04.014.

13. Mousavi SR, Moradi A, Sobhiyeh MR, Jabbehdari S, Azimi B, Lotfollahzadeh S, et al. A patient with cystic lymphangioma in pancreas. Gastroenterol Hepatol Bed Bench 2013;6:159-164. doi.

14. Kambakamba P, Lesurtel M, Breitenstein S, Emmert M, Wilhelm M, Clavien P. Giant mesenteric cystic lymphangioma of mesocolic origin in an asymptomatic adult patient. J Surg Case Rep 2012;2012:4. doi: $10.1093 / \mathrm{jscr} / 2012.6 .4$.

\section{Figures}




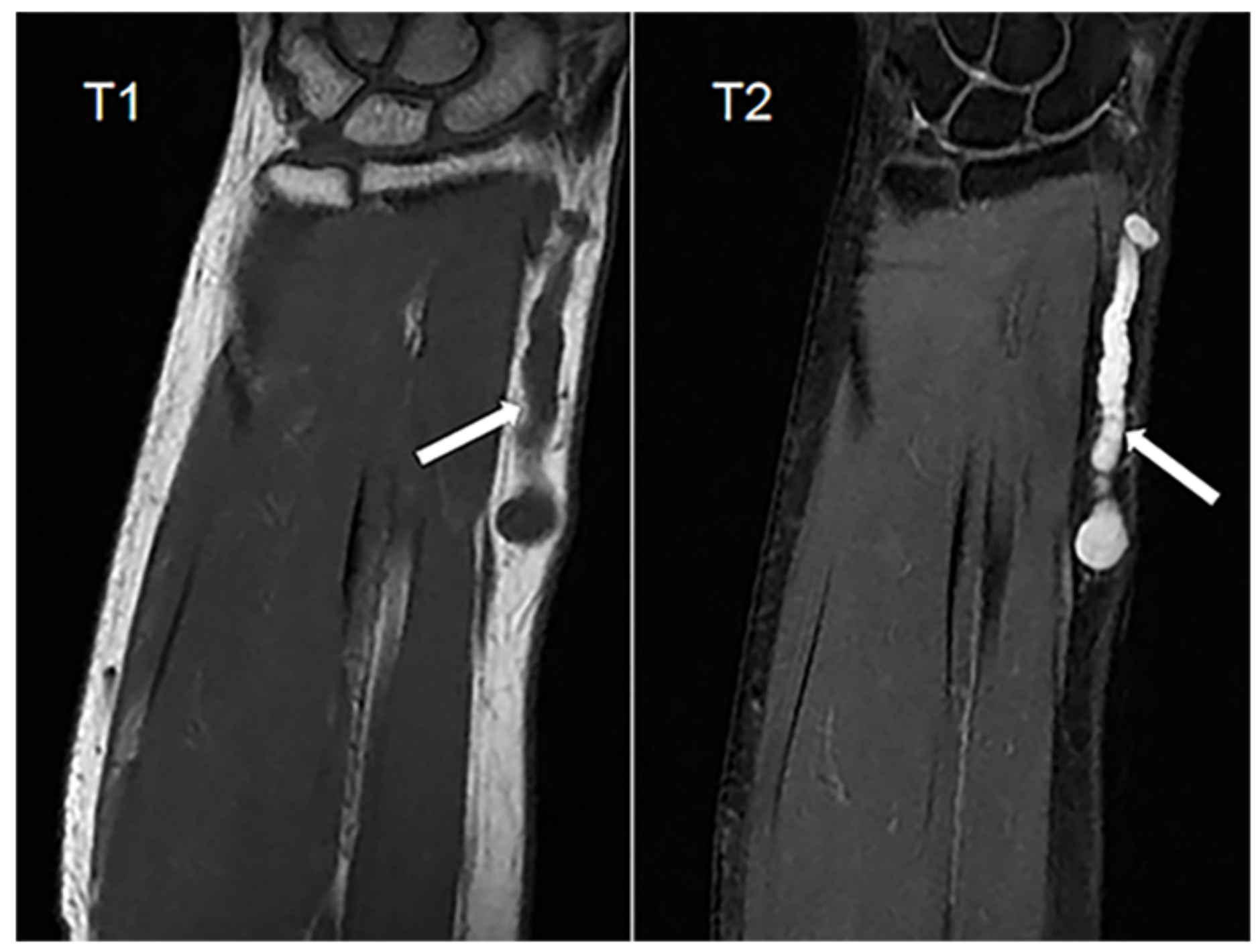

\section{Figure 1}

Magnetic resonance imaging showing isointense or hypointense signal relative to muscle signal on T1weighted imaging (WI); hyperintense signal on T2WI. 


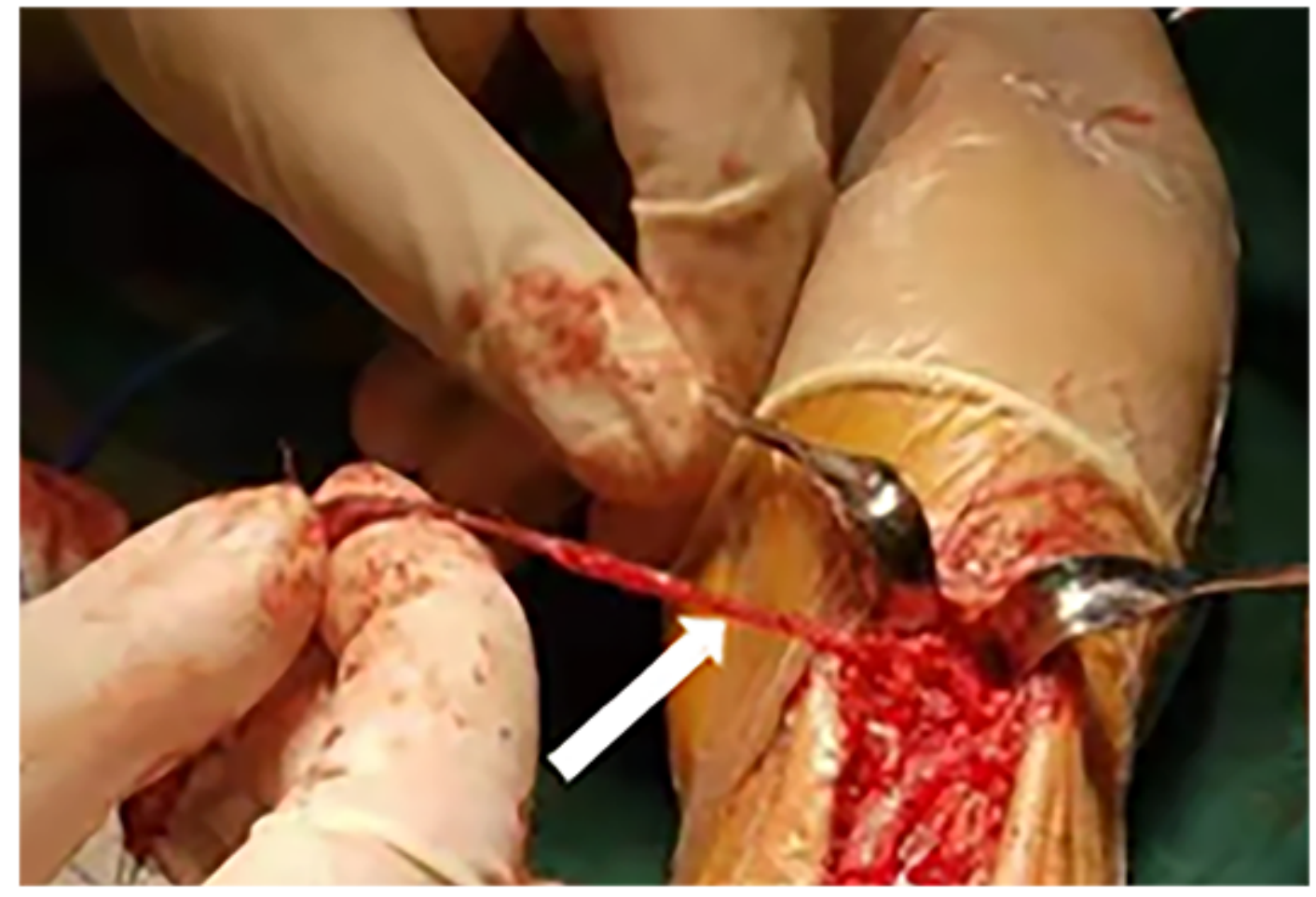

Figure 2

Intraoperative image showing dissection of the mass. The skin is cut above the radial side of the right forearm, which reveals an approximately $10-\mathrm{cm}$ long cord-like mass.
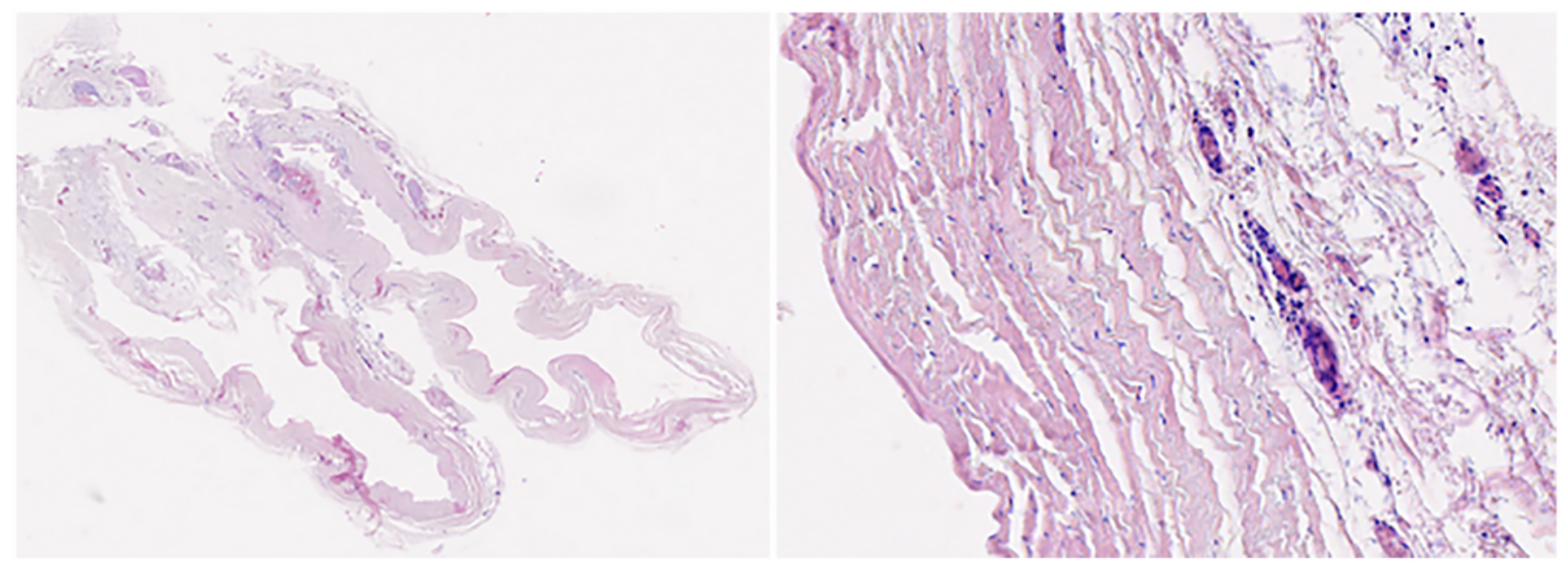

\section{Figure 3}

H\&E findings showing a dilated cystic space and a cystic wall containing flattened endothelial cells, regular smooth muscle fascicles, small adipocytes, and mature lymphocytes. 


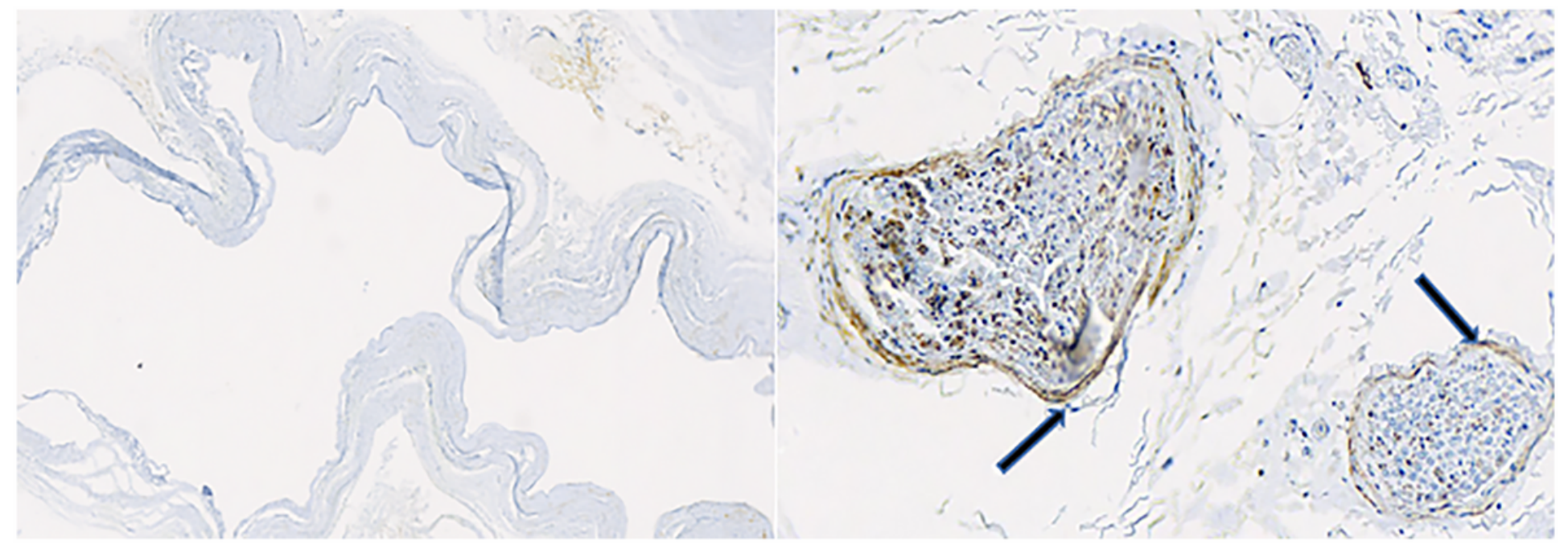

\section{Figure 4}

IHC findings showing the lumen of lymphatic vessels clearly outlined by D2-40 immunostaining in brown in the specimen. 